\title{
Spectrométrie de masse dans les maladies métaboliques de l'enfant
}

La spectrométrie de masse permet d'identifier et de mesurer avec précision des composés chimiques au sein de mélanges complexes. Elle trouve ainsi plusieurs applications dans les maladies héréditaires de l'enfant qui se traduisent par l'accumulation de métabolites normalement présents seulement à l'état de traces. En identifiant la nature chimique d'un ou plusieurs métabolites anormalement présents, elle permet de localiser le bloc enzymatique en amont duquel s'accumulent ces produits. Par sa spécificité et sa sensibilité, elle permet également de quantifier ces métabolites et trouve ainsi une application directe au conseil génétique et au diagnostic anténatal.

\section{Francis Rocchiccioli}

Remerciements

Je dédie cet article à la mémoire du professeur Pierre Cartier. Je remercie Pierre Bougnères et Patrick Aubourg pour leur collaboration et Nathalie Lepetit et Michel Auclair pour leur aide technique.

\section{ADRESSE}

F. Rocchiccioli : chef de travaux des universités, praticien hospitalier. Inserm U.188 et laboratoire de biochimie, hôpital Saint-Vincent-de-Paul, 74, avenue Denfert-Rochereau, 75674 Paris Cedex 14, France.

$\mathrm{m} / \mathrm{s} n^{\circ} 9$ vol. 5 , novembre 89 a plupart des maladies héréditaires dues à un déficit enzymatique interrompant une voie biochimique se traduisent par l'accumulation d'intermédiaires du métabolisme qui peut être mise en évidence, selon les cas, dans les milieux biologiques (plasma, urine, liquide céphalorachidien, liquide amniotique) ou dans les cellules de certains tissus (fibroblastes, trophoblastes, foie, muscle). Cependant, même lorsque cette accumulation est majeure (100 à 1000 fois la normale), les échantillons biologiques à visée diagnostique ne contiennent le plus souvent que quelques centaines de nanogrammes des produits caractérisant la maladie, d'où la nécessité d'utiliser une méthode capable de concilier une grande spécificité pour l'identification de ces molécules et une grande sensibilité pour leur détection et leur quantification précise.

Le couplage chromatographie en phase gazeuse-spectrométrie de masse est devenu l'outil par excellence pour l'analyse de molécules organiques [1-3]. Ses performances sont liées au traitement par l'informatique des données fournies par une méthode de séparation, la chromatographie en phase gazeuse, et une méthode d'identification, la spectrométrie de masse. Il est ainsi possible de détecter et de mesurer de toutes petites quantités de produits «anormaux » au sein de mélanges complexes. La spectrométrie de masse trouve ainsi plusieurs applica- 
tions dans le domaine des maladies métaboliques de l'enfant: (1) en identifiant la nature chimique d'un ou plusieurs composé(s) accumulé(s) situé(s) en amont d'un bloc enzymatique, elle permet de localiser l'étape déficiente ; (2) par sa spécificité et sa sensibilité, la spectrométrie de masse permet de quantifier avec grande précision certains métabolites dont la mesure est difficile voire impossible avec d'autres méthodes (chromatographie en phase gazeuse, chromatographie liquide haute performance). Nous présentons ici quelques applications médicales illustrant l'intérêt de la spectrométrie de masse dans le domaine des maladies métaboliques de l'enfant.

\section{De la molécule au spectre de masse}

L'analyse par spectrométrie de masse d'un produit organique au sein d'un milieu biologique nécessite un traitement de l'échantillon comportant : (1) une purification (déprotéinisation, chromatographie par échange d'ions); (2) une extraction à l'aide d'un solvant organique (éther, acétate d'éthyle) qu'il est ensuite possible d'éliminer par évaporation et (3) une dérivation par un réactif qui transforme chimiquement les fonctions polaires de la molécule (acide, amine et/ou hydroxyle) en groupements apolaires (figure 1).

La séparation des molécules est réalisée par chromatographie en phase gazeuse et les molécules éluées de la colonne chromatographique diffusent ensuite directement dans la source ou chambre du spectromètre de masse où a lieu leur ionisation par impact électronique ou ionisation chimique (figure 2).

L'ionisation par impact électronique se produit par arrachement d'un électron périphérique de la molécule et obtention d'une espèce ionique instable à nombre impair d'électrons, l'ion moléculaire, qui se fragmente en ions de plus faibles masses $m_{1}$, $m_{2}, \ldots$ (figure 2) par ruptures simples de liaisons et/ou réarrangements moléculaires, ce qui fournit autant d'espèces ioniques. C'est l'ensemble de ces familles d'ions (figure 2) qui constitue le spectre de masse de la molécule. Le processus de fragmen-

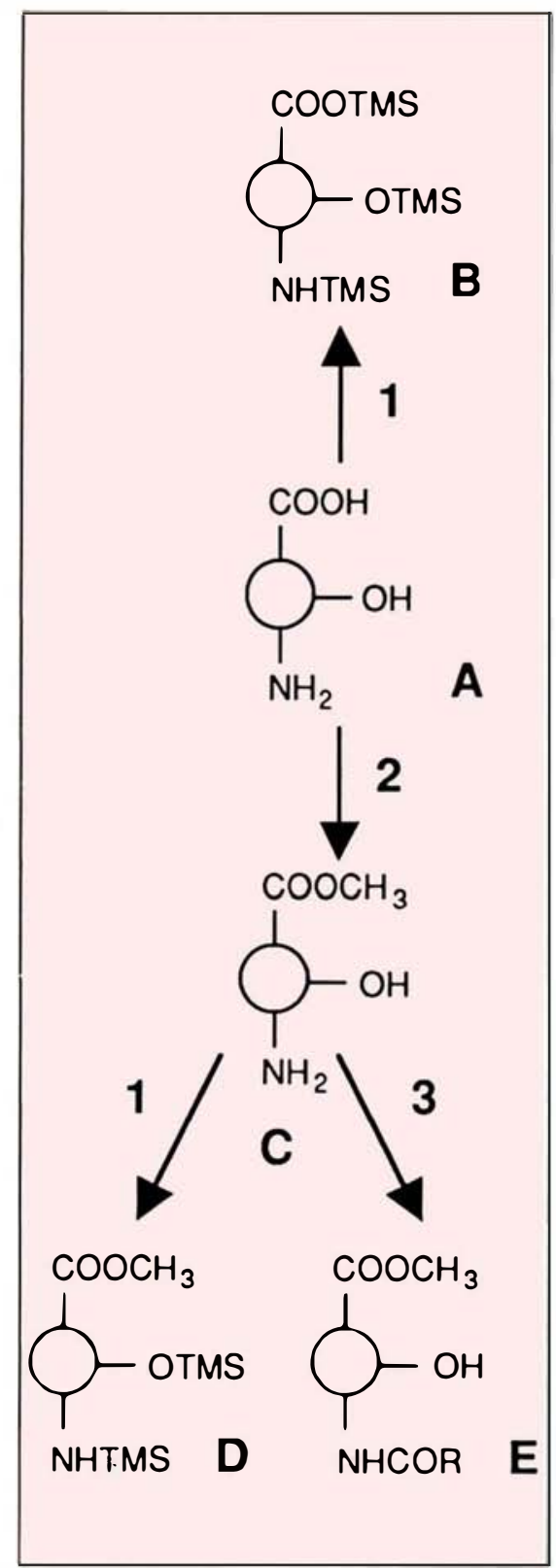

Figure 2. Schéma d'obtention d'un spectre de masse dans un couplage chromatographie en phase gazeuse-spectrométrie de masse. La molécule $M$, éluée sous forme gazeuse de la colonne chromatographique, est ionisée par un faisceau d'électrons (70 eV) avec formation de l'ion moléculaire $\mathrm{M}^{+}$. instable qui se fragmente en ions de plus faibles masses séparés au niveau du quadripôle.
Figure 1. Principales dérivations de molécules organiques extraites d'un milieu biologique. La molécule organique extraite d'un milieu biologique est schématisée en $A$; elle comporte une ou plusieurs fonctions polaires dont les plus fréquemment rencontrées sont un carboxyle ( $\mathrm{COOH})$, une amine $\left(\mathrm{NH}_{2}\right)$ ou un hydroxyle $(\mathrm{OH})$. B est obtenu par addition d'un réactif (1) donneur de groupes triméthylsilyl (TMS). C est obtenu par chauffage en présence de méthanol chlorhydrique anhydre (2) ou par addition de diazométhane. $D$ est obtenu par addition du réactif(1) à $C$. $E$ est obtenu par traitement de $C$ par un anhydride d'acide (3) (acétique, $R=$ $\mathrm{CH}_{3} ;$ trifluoroacétique, $\mathrm{R}=\mathrm{CF}_{3}$ ).

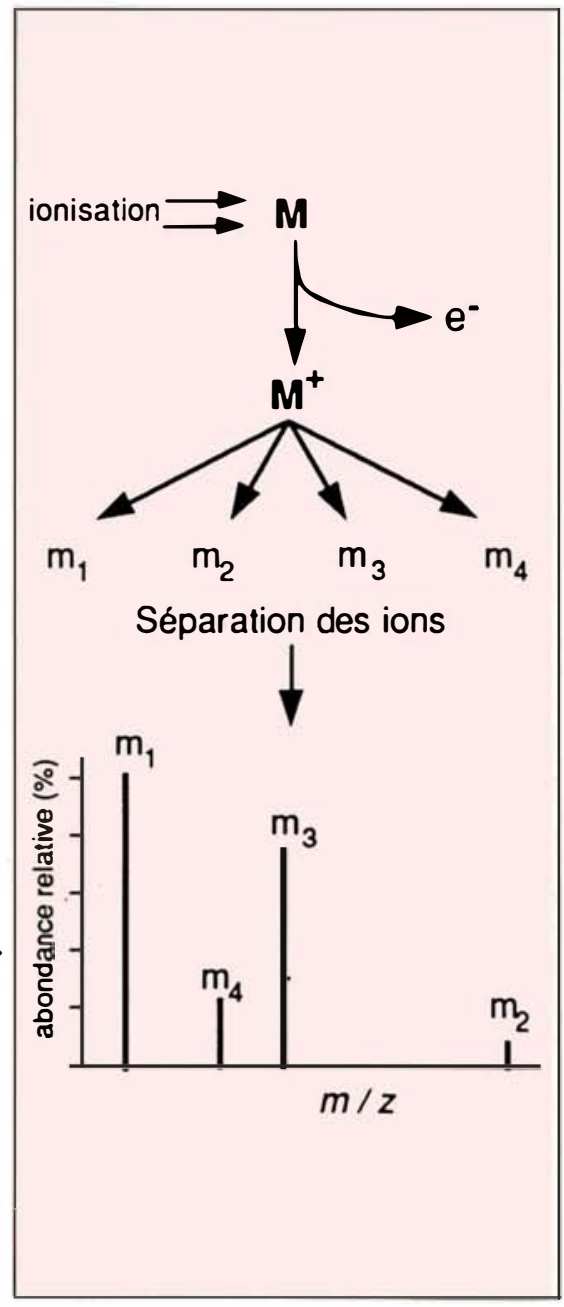

$\mathrm{m} / \mathrm{s} n^{\circ} 9$ vol. 5, novembre 89 


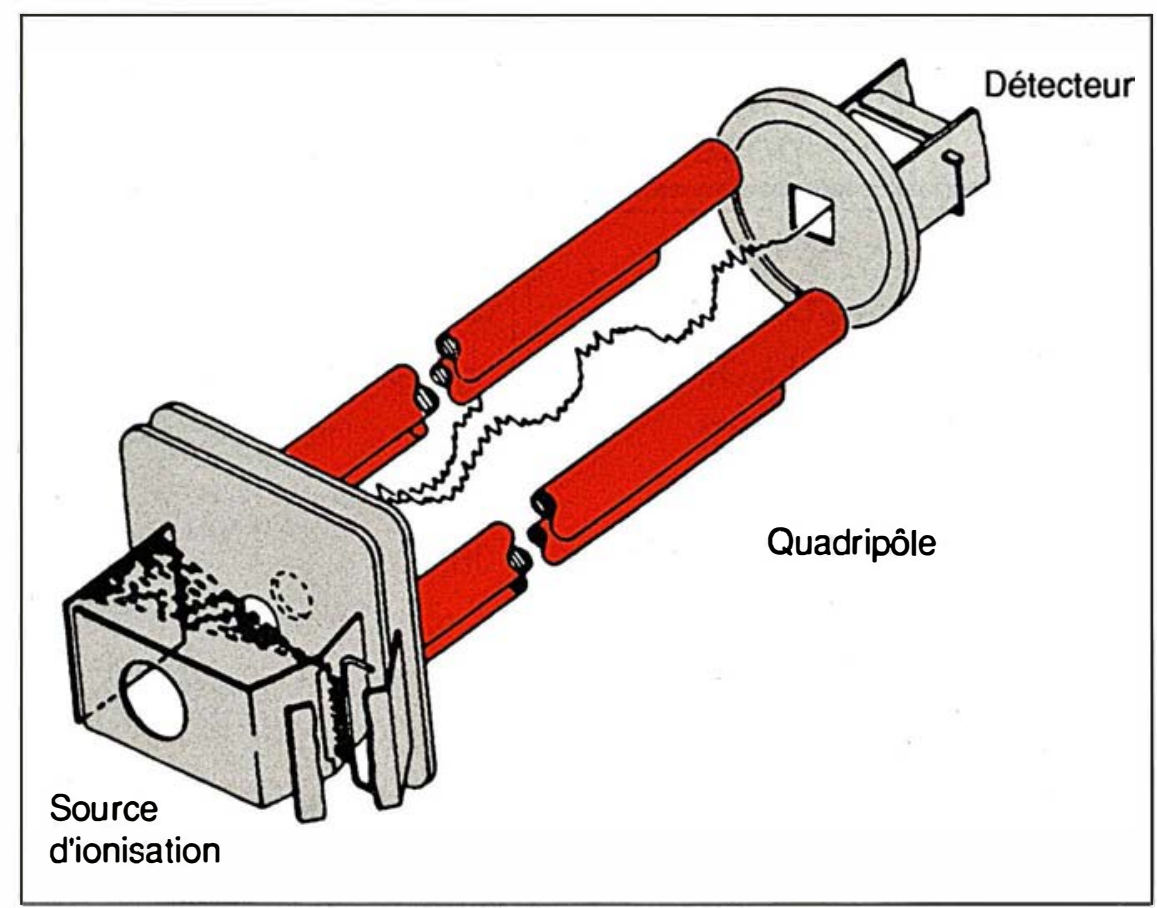

Figure 3. Détail du quadripôle placé dans une enceinte sous un vide de $10.6 \mathrm{mmHg}$. Les ions formés dans la source sont soumis à l'action conjuguée d'un champ électrique alternatif (radiofréquence) et d'un champ électrique continu produit entre deux paires de barres conductrices isolées, connectées deux à deux, en carré, à des générateurs de tensions continues et alternatives d'amplitude variable. Une paire de barres est alimentée avec un voltage continu positif et un voltage alternatif; l'autre paire par un voltage continu identique mais négatif et un voltage alternatif en discordance de phase de $180^{\circ}$. On produit entre ces barres rigoureusement alignées un champ électrostatique qui fait osciller les ions. Suivant les amplitudes respectives des tensions continues et alternatives, la fréquence, la distance entre les barres et leur formes, seuls les ions d'un rapport $\mathrm{m} / \mathrm{z}$ déterminé suivent une trajectoire limitée et stable et parcourent le quadripôle pour atteindre le détecteur. Les autres espèces ioniques ont des amplitudes d'oscillations trop grandes et sont collectées, déchargées sur les barres conductrices et pompées sous vide. L'impact des ions sur les barres du quadripôle peut donner lieu à l'émission de rayons $X$ de faibles énergies. Les éléments neutres excités qu'ils peuvent induire doivent être éliminés avant de parvenir au niveau du détecteur : c'est la raison pour laquelle on défocalise légèrement celui-ci. Le spectre de masse complet s'obtient en faisant osciller à grande fréquence l'amplitude des tensions continue et alternative tout en maintenant leur rapport d'intensité constant.

chimique de la molécule et les fragments ainsi observés en constituent en quelque sorte une «empreinte digitale ». L'ion moléculaire est dans la majorité des cas tellement instable qu'il n'est pas détecté.

Lorsque l'on utilise l'ionisation chimique, les ions sont formés par des réactions entre un gaz réactionnel (ammoniac, méthane) à haute pression $\left(10^{-2}\right.$ à $\left.10^{-1} \mathrm{mmHg}\right)$ et les molécules non chargées du composé à analyser. L'ionisation se produit dans en ionisation chimique car il correspond à la focalisation de tous les fragments observés en impact électronique sur un ion unique rendant ce signal d'autant plus intense. Le gain dans la sensibilité de détection est alors d'un facteur $10^{4}$. Par ailleurs, la spécificité est d'autant plus augmentée que le signal observé en ionisation chimique correspond à la molécule entière.

Les familles d'ions formés dans la source d'ionisation sont ensuite extraits, focalisés et accélérés par des lentilles successives de façon à obtenir un faisceau ionique dont toutes les particules ont la même énergie cinétique.

L'analyse des familles d'ions est réalisée selon le rapport de leur masse à leur charge $(\mathrm{m} / \mathrm{z})$ dans un filtre quadripolaure (figure 3). Les signaux obtenus sont amplifiés par un photomultiplicateur d'électrons qui enregistre aussi la collection séquentielle des faisceaux ioniques en donnant un signal dont l'intensité est proportionnelle à la quantité d'ions qui arrivent sur la première dynode. Les valeurs des rapports $m / z$ sont proportionnelles aux amplitudes des voltages enregistrés et sont calculées par rapport à un calibrage électronique préalable réalisée au moyen de composés de référence présentant des espèces ioniques connues dans toute la gamme des masses étudiées (perfluorokérosènes).

L'acquisition et le traitement des données provenant du spectomètre de masse nécessitent l'utilisation d'un ordinateur qui intervient à plusieurs niveaux: (a) contrôle des principales fonctions de l'instrument; (b) sélection des paramètres d'enregistrement ; (c) acquisition des données transmises par le spectomètre : spectres de masse, courant ionique total, temps de rétention, intensités des pics,...; (d) traitement des informations (recherche en librairie, détection d'ions sélectionnés, calcul de droite d'étalonnage, calcul de concentrations, ...).

une ou plusieurs espec(s) ionique(s) u plusieurs espèce(s) ionique(s) par réactions ions-molécules. Ces ions rencontrent ensuite les molécules de l'échantillon (M) et leur transfèrent un proton, entraînant la formation de l'ion «quasi-moléculaire » $[\mathrm{MH}+]$, à nombre pair d'électrons, donc stable et en règle générale le plus abondant du spectre de masse

\section{Du spectre de masse à la molécule}

Identifier des composés anormaux dans les urines ou le sérum d'un enfant présentant des signes, aigus (coma) ou chroniques, d'une maladie 


\section{RÉFÉRENCES}

1. De Graeve J, Berthou F, Prost M. Méthodes chromatographiques couplées à la spectrométrie de masse. Paris : Masson, 1986.

2. Watson JT. Introduction to mass spectrometry ( $2^{\text {nd }}$ éd.). New York : Raven Press, 1985.

3. Hill RE, Whelan DT. Mass spectrometry and clinical chemistry. Clin Chim Acta 1984 139: 231-94.

4. Rocchiccioli F, Cartier PH, Bougnères PF Mass spectrometric identification of abnormal aromatic compounds in the urine of a child with Reye's like syndrome. Biomed Mass Spectrom 1984; 11 : 127-31.

5. T joa SS, Fennessey PV. Acylglycines. The gas chromatograph-mass spectrometric identification and interpretation of their spectra. Clin Chim Acta 1979 ; 95 : 35-45.

6. Poll The BT, Vamecq J, Draye JP, Saudubray JM. Un nouveau groupe d'erreurs innées du métabolisme : les maladies peroxysomiales. médecine/sciences 1988 ; 4 : 553-9.

7. Rocchiccioli F, Aubourg P, Bougnères PF Medium- and long-chain dicarboxylic aciduria in patients with Zellweger syndrome and neonatal adrenoleukodystrophy. Pediatr Res $1986 ; 20: 62-6$

8. Chalmers RA, Lawson AM. Organic acids in man, the analytical chemistry, biochemis try and diagnosis of the organic acidurias. London - New York: Chapman and Hall 1982 : 466-7.

9. Rhead WJ, Amendt BA, Fritchman KS Felts SJ. Dicarboxylic aciduria : deficient l${ }^{14} \mathrm{C}$ octanoate oxidation and medium-chain acyl-CoA dehydrogenase in fibroblasts. Science 1983 ; 221 : 73-5.

10. Bougnères PF, Rocchiccioli F, Kolvraa $S$, et al. Medium-chain acyl-CoA dehydrogenase deficiency in two siblings with a Reye-like syndrome. J Pediatr 1985; 106 : 918-21.

11. Gregersen N. Mortensen PB, Kolvraa S On the biological origin of $\mathrm{C}_{6}-\mathrm{C}_{10}$-dicarboxy-

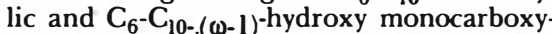
lic acids in human and rat with acyl-CoA dehydrogenation deficiencies : in vitro studies on the $\omega$ - and ( $\omega$-1)-oxidation of mediumchain $\left(\mathrm{C}_{6}-\mathrm{C}_{12}\right)$ fatty acids in human and rat liver. Pediatr Res 1983; 12 : 828-34

12. Rinaldo P, O'Shea JJ, Coates PM, Hale DE, Stanley CA, Tanaka K. Medium-chain acyl-CoA dehydrogenase deficiency. Diagnosis by stable-isotope dilution measurement of urinary n-hexanoylglycine and 3-phenylpropionylglycine. $N$ Engl J Med 1988; 319 :

\begin{tabular}{|c|c|c|}
\hline $\mathbf{m} / \mathbf{z}$ & $\begin{array}{l}\text { Formule } \\
\text { possible }\end{array}$ & Composés \\
\hline 26 & $\mathrm{C}_{2} \mathrm{H}_{2}$ & hydrocarbures \\
\hline 28 & $\mathrm{CO}^{2}$ & carbonyle \\
\hline 31 & $\mathrm{CH}_{2}=\mathrm{OH}$ & alcool primaire \\
\hline $35 / 37$ (3:1) & ${ }^{35} \mathrm{Cl} /{ }^{37} \mathrm{Cl}$ & dérivés chlorés \\
\hline 43 & $\mathrm{CH}_{7}$ & hydrocarbures \\
\hline 43 & $\mathrm{CH}_{3} \mathrm{CO}$ & $\mathrm{CH}_{3} \mathrm{CO}-\mathrm{X}$ \\
\hline 45 & $\mathrm{CH}_{3}-\mathrm{CH}=\mathrm{OH}$ & alcool secondaire \\
\hline $55^{*}$ & $\mathrm{C}_{4} \mathrm{H}_{7}$ & hydrocarbures insaturés \\
\hline $57^{*}$ & $\mathrm{C}_{4} \mathrm{H}_{9}$ & hydrocarbures \\
\hline $73^{*}$ & $\mathrm{Si}\left(\mathrm{CH}_{3}\right)_{3}$ & dérivés triméthylsilylés \\
\hline $74^{\star}$ & $\mathrm{CH}_{2}=\mathrm{C}(\mathrm{OH}) \mathrm{OCH}_{3}$ & $\begin{array}{l}\text { esters méthyliques d'acides } \\
\text { gras ayant un hydrogène en } \gamma \\
\text { (réarrangement de McLafferty) }\end{array}$ \\
\hline $77^{*}$ & $\mathrm{C}_{6} \mathrm{H}_{5}$ & dérivés aromatiques \\
\hline $79 / 81(1: 1)$ & ${ }^{79} \mathrm{Br} /{ }^{-1} \mathrm{Br}$ & dérivés bromés \\
\hline $91^{*}$ & $\mathrm{C}_{7} \mathrm{H}_{7}$ & dérivés aromatiques \\
\hline 103 & $\mathrm{C}_{6} \mathrm{H}_{5} \mathrm{CH}=\mathrm{CH}$ & dérivés aromatiques \\
\hline $105^{*}$ & $\mathrm{C}_{6} \mathrm{H}_{5} \mathrm{CO}$ & cétone en $\alpha$ d'un benzène \\
\hline 129 & $\mathrm{C}_{5} \mathrm{H}_{9} \mathrm{O}_{2} \mathrm{Si}$ & acides gras triméthylsilylés \\
\hline $147^{*}$ & $\mathrm{C}_{5} \mathrm{H}_{15} \mathrm{OSi}_{2}$ & dérivés polytriméthylsilylés \\
\hline $149^{*}$ & $\mathrm{C}_{8} \mathrm{H}_{5} \mathrm{O}_{3}$ & phtalates \\
\hline
\end{tabular}

Figure 4. Structure des fragments de basses masses. La valeur des fragments de faibles masses est d'une importance diagnostique capitale pour l'interprétation des spectres de masses en impact électronique. Les fragments de $\mathrm{m} / \mathrm{z}$ $>50$ unités de masse atomique sont toujours très intenses et même très souvent les pics de base des spectres de masse. Le spectre obtenu est automatiquement normalisé par rapport au fragment le plus intense (pic de base).

métabolique est l'étape diagnostique majeure. L'identification d'une molécule organique inconnue peut être réalisée à partir de l'analyse des fragments de son spectre de masse, la structure de la molécule originelle étant reconstituée comme un puzzle par déduction en suivant un raisonnement par étapes: (1) identifier l'ion moléculaire $\mathrm{M}$, dont la valeur sert de point de départ pour l'étude du processus de fragmentation; (2) si l'ion moléculaire n'est pas détectable, l'identifier par dérivation du produit (triméthylsilylation par exemple) et/ou en ionisation chimique; (3) noter la parité de l'ion moléculaire : toute molécule organique qui a un poids moléculaire impair renferme en effet un nombre 


\begin{tabular}{|c|c|}
\hline $\begin{array}{l}\text { Fragment } \\
\text { à } \mathbf{m} / \mathbf{z}[M-x]\end{array}$ & $\begin{array}{l}\text { Famille } \\
\text { de molécules }\end{array}$ \\
\hline$[M-1]$ & Aldéhyde, acétals \\
\hline$[M-15]$ & esthers méthyliques, acétal, dérivés triméthylsilylés \\
\hline$[M-16]$ & Composés nitrés aromatiques, $\mathrm{N}$-oxydes, amides aromatiques \\
\hline$[\mathrm{M}-17]$ & Acides carboxyliques non dérivés \\
\hline$[M-18]$ & $\begin{array}{l}\text { aldéhyde aliphatique(linéaires), alcools primaires, } \\
\text { éthers aliphatiques, acides carboxyliques }\end{array}$ \\
\hline$[M-31]$ & esters méthyliques, dérivés méthoxylés \\
\hline$[M-34]$ & thiols \\
\hline$[M-42]$ & acétates \\
\hline$[M-59]$ & ester méthyliques, ester propyliques \\
\hline$[M-117]$ & acides $\alpha$-hydroxylés triméthylsilylés \\
\hline$[M-131]$ & $\begin{array}{l}\text { acylglycines triméthylsilylées } \\
\text { acides } \beta \text {-hydroxylés triméthylsilylés }\end{array}$ \\
\hline$[M-146]$ & acylglycines triméthylsilylées \\
\hline
\end{tabular}

Figure 5. Identification des familles de molécules à partir des fragments perdus de masse $X$ provenant de l'ion moléculaire $M$. La perte de fragments neutres, provenant directement de l'ion moléculaire, permet d'identifier des fonctions chimiques présentes au sein des molécules, donnant ainsi des informations complémentaires à celles précédemment obtenues (voir figure 4).

impair d'atomes d'azote puisque sa valence est impaire (règle de l'azote); (4) repérer les fragments de faibles masses (figure 4) ainsi que la perte de fragments neutres provenant de l'ion moléculaire (figure 5), ce qui permet d'identifier certains groupements chimiques au sein des molécules.

A titre d'exemple, nous montrerons la démarche nécessaire à l'identification d'un composé inconnu présent dans les urines d'un enfant lors d'un coma métabolique. Ce produit triméthylsilylé correspond au composé $\mathrm{X}$ de la figure 6, p. 650 dont le spectre de masse est présenté en encart [4]. Les dérivés triméthylsilylés se caractérisant toujours entre autres par la perte d'un méthyle (figure 5), (figure 4), et fragments différant de 14 ou 28 unités de masse atomique: 158,172 et 200 d'une part, 189 et 217 d'autre part. L'ensemble de ces données conduit à la formulation d'un schéma de fragmentation correspondant à la structure de la subérylglycine. Cette hypothèse a été confirmée par hydrolyse chimique de ce composé et caractérisation de l'acide subérique (acide dicarboxylique linéraire à huit atomes de carbone) libéré. La preuve finale est apportée par confrontation du spectre de masse du produit inconnu avec celui de la subéryl-glycine de synthèse, et par coïncidence de leurs caractéristiques (temps de rétention) en chromatographie en phase gazeuse.

Un autre exemple est l'identification d'un composé inconnu présent dans les urines d'un patient atteint d'un syndrome de Zellweger, maladie caractérisée par un défaut des fonctions peroxysomiales [6]. Ce dérivé triméthylsilylé corespond au produit inconnu $\mathrm{Y}$ de la figure 7 [7]. Le fragment de plus haute masse visible à $\mathrm{m} / \mathrm{z} 419$ (figure 7) est compatible avec un poids moléculaire de 434 (figure 5), confirmé par ionisation chimique $\left[\mathrm{MH}^{+}=435\right]$. Le fragment à $\mathrm{m} / \mathrm{z} 147$ est caractéristique d'un dérivé polytriméthylsilylé (figure 4) L'ion intense à $\mathrm{m} / \mathrm{z} 317$ [M - 117] peut correspondre à un dérivé d'acide gras hydroxylé en $\alpha$, puisque ce type de fragmentation se retrouve également dans des composés triméthylsilylés plus simples comme l'acide lactique ou l'acide hydroxy-2 butyrique [8]. Le fragment à $\mathrm{m} / \mathrm{z} 391$ [M - 43] peut correspondre à la perte d'un carbonyle $(\mathrm{CO}=28)$ à partir de l'ion à $\mathrm{m} / \mathrm{z} 419$ [M - 15]. Cette hypothèse est également en accord avec la structure d'un acide $\alpha$-hydroxylé, par analogie avec la fragmentation des acides $\beta$-hydroxylés qui perdent un méthylène-carbonyle $\left(\mathrm{CH}_{2} \mathrm{CO}=42\right)$ à partir du [M - 15]. Enfin, le fragment à $\mathrm{m} / \mathrm{z} 301$ provient de la migration, sur le côté opposé de la molécule, d'un groupe triméthylsilyle de l'ion à $\mathrm{m} / \mathrm{z} 317$ avec perte simultanée d'un atome d'oxygène. L'hypothèse de cette structure, celle d'un acide dicarboxylique à dix atomes de carbone présentant une fonction hydroxyle en $\alpha$ d'un des deux carboxyles, est fortifiée par le comportement chro- 


\section{RÉFÉRENCES}

13. Aubourg P, Robain O, Rocchiccioli F Dancea S, Scotto J, The cerebro-hepato-renal (Zellweger) syndrome : lamellar lipid profiles in adrenocortical, hepatic mesenchymal, astrocyte cells and increased levels of very long chain fatty acids and phytanic acid in the plasma. J Neurol Sci $1985 ; 69: 9-25$.

14. Chalmers RA, Spellacy E. A method for the pre- and post-natal detection of defects of isovalerate metabolism. Clin Sci 1979 ; 57 : 25

15. Sweetman L, Weyler W, Shafai T, Young PE, Nyhan WL. Prenatal diagnosis of propionic acidemia. JAMA 1979 ; 242 : 1048 52.

16. Zinn $\mathrm{AB}$, Hine $\mathrm{DG}$, Mahoney $\mathrm{MJ}$, Tanaka $K$. The stable isotope dilution method for measurement of methylmalonic acid : a highly accurate approach to the prenatal diagnosis of methylmalonic acidemia Pediatr Res 1982 ; 16 : 740-5.

17. Goodman SI, Gallegos DA, Pullin CJ, et al. Antenatal diagnosis of glutaric aciduria. Am J Hum Genet 1980 ; 32 : 695-9.

18. Mitchell G, Saudubray JM, Benoit Y, et al. Antenatal diagnosis of glutaric aciduria type II. Lancet 1983 ; i : 1099.

19. Jakobs C, Kvittingen EA, Berger R, Haagen $\mathbf{A}$, Kleijer $\mathbf{W}$, Niermeijer $\mathbf{M}$. Prenatal diagnosis of tyrosinaemia type I by use of stable isotope dilution mass spectrometry. Eur J Pediatr 1985 ; 144 : 209-10.

20. Solish GI, Moser HW, Ringer LD, Moser AE, Tiffany C, Schutta E. The prenatal diagnosis of the cerebro-hepato-renal syndrome of Zellweger. Prenat Diagn 1985 ; 5 : 27-34.

21. Rocchiccioli F, Aubourg P, Choiset A Immediate prenatal diagnosis of Zellweger syndrome by direct measurement of very long chain fatty acids in chorionic villus cells Prenat Diagn 1987 ; 7 : 349-54

22. Moser HW, Moser AE, Trojak JE, Sup plee SW. Identification of female carriers of adrenoleukodystrophy. J Pediatr 1983 ; 103 . 54-9.

23. Beavis RC, Chait BT. Factors affecting the ultraviolet laser desorption of proteins. Rapid Commun Mass Spectrom 1989; 3 : 233-

24. Roe CR, Millington DS, Maltby DA Bohan TP, Kahler SG, Chalmers RA. Dia gnostic and therapeutic implications of medium-chain acylcarnitines in the medium-

chain acyl-CoA dehydrogenase deficiency.

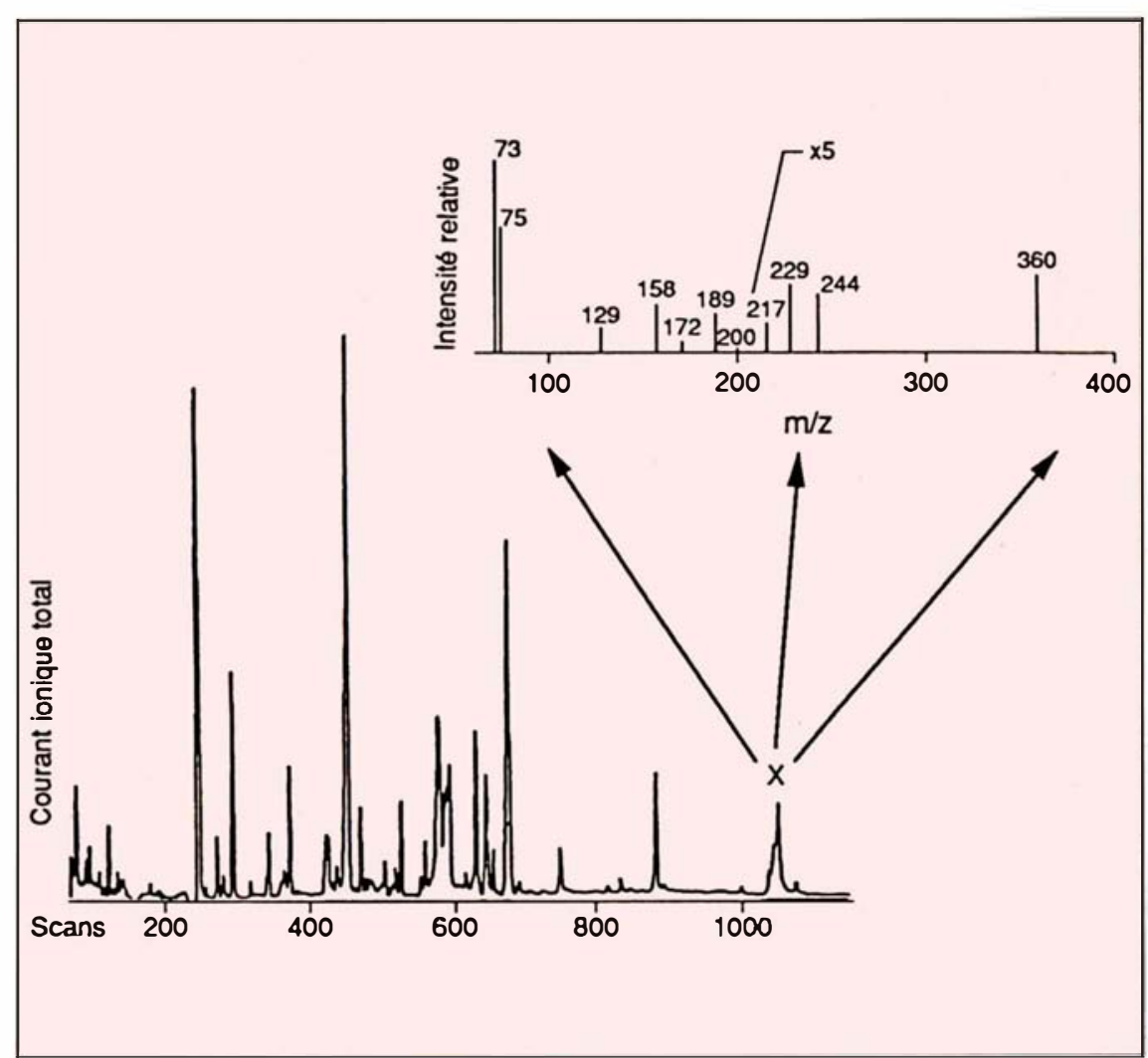

Figure 6. Profil des acides organiques extraits de l'urine recueillie au cours d'un coma hypoglycémique chez un patient présentant une déficience en déshydrogénase des acyl-CoA à chaîne moyenne. La séparation est réalisée sur une colonne capillaire apolaire. Le "Scan » correspond à l'unité de temps d'enregistrement des informations (de l'ordre de 0,5 seconde), chaque scan étant stocké indépendamment des autres et pouvant être visualisé séparément par le traitement de données. Le profil chromatographique ou courant ionique total est reconstitué automatiquement par la somme de tous les ions formés. Chaque pic chromatographique correspond à un produit distinct. Les spectres de masse des différents produits sont obtenus par traitement informatique. A titre d'exemple, le spectre de masse du composé $X$ (subéryl-glycine), un des marqueurs de la maladie, est présenté en encart.

matographique de ce produit qui est élué juste avant son isomère, l'acide hydroxy-3-sébacique (produit $Z$ de la figure 7). En effet, les acides hydroxylés et triméthylsilylés sont élués d'autant plus tardivement que l'hydroxyle est plus éloigné de la fonction carboxylique. La preuve finale est apportée par superposition du spectre de masse du produit inconnu avec celui de l'acide hydroxy-2-sébacique authentiquement pur.

L'identification d'une substance par couplage chromatographie en phase gazeuse-spectrométrie de masse ne peut être certaine que dans la mesure où les spectres de masse et les com- portements chromatographiques du produit à identifier et du produit authentiquement pur sont rigoureusement superposables.

\section{Du métabolite au déficit enzymatique}

Les maladies qui touchent la $\beta$-oxydation des acides gras représentent un exemple parmi d'autres où l'analyse des métabolites urinaires anormaux s'intègre dans un schéma physiopathologique cohérent permettant de caractériser le déficit enzymatique. L'une de ces maladies, le déficit en déshydrogénase des acylCoA à chaîne moyenne [9], se carac- 


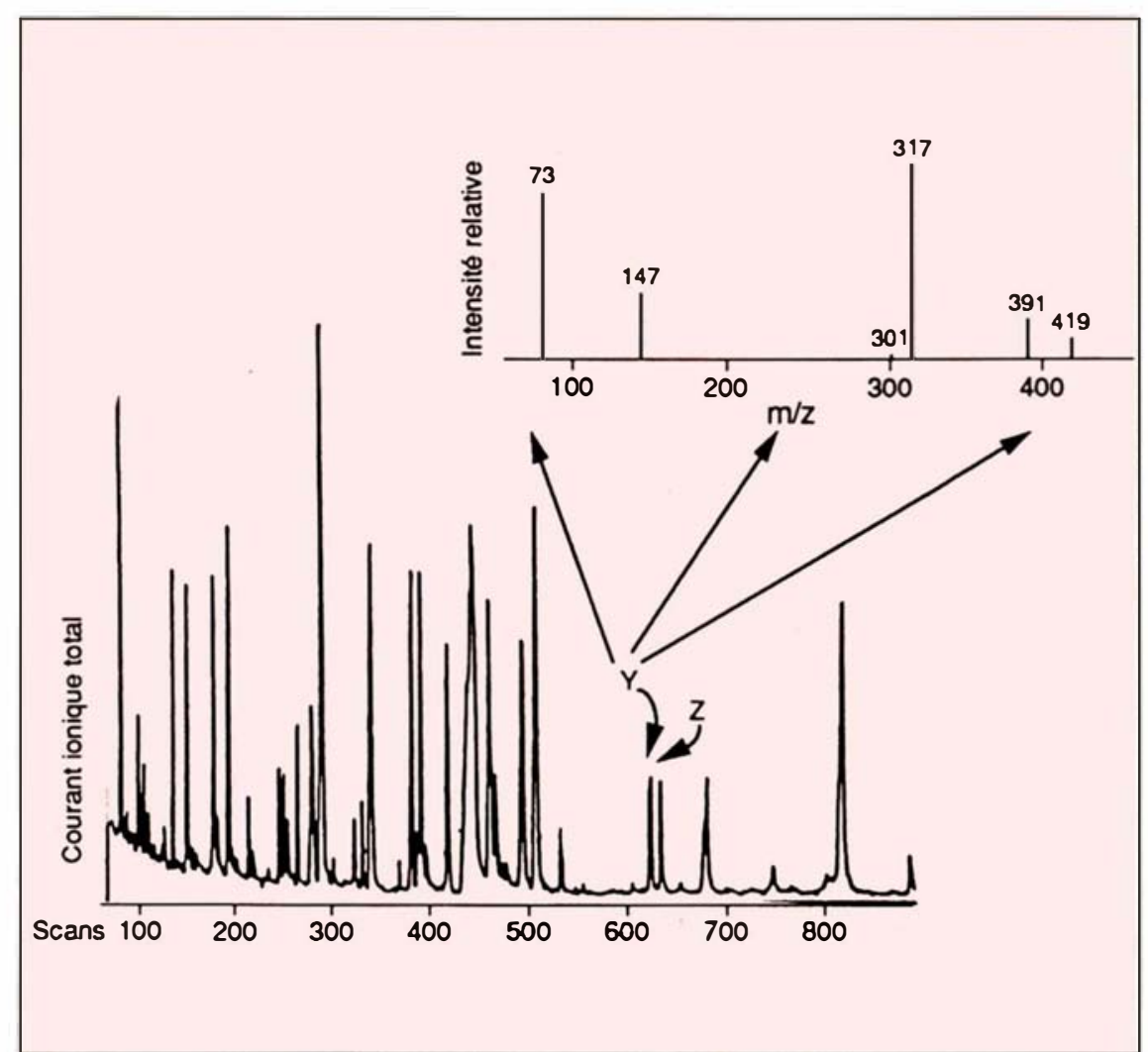

Figure 7. Profil des acides organiques extraits d'une urine d'un patient présentant un syndrome de Zellweger. Le spectre de masse du composé $Y$ (acide hydroxy-2-sébacique) est présenté en encart. Le composé $Z$ correspond à l'acide hydroxy-3-sébacique.

térise par un profil particulier de métabolites urinaires $[4,10]$ (figure 6$)$ parmi lesquels des acides dicarboxyliques à chaîne moyenne, des acides hydroxylés en $(\omega-1)$ et des conjugués de la glycine, la subéryl- et la phénylpropionyl-glycine.

Dans le cas rapporté ici, le patient présentait une acidurie dicarboxylique majeure dont le profil est commun à l'ensemble des maladies dues à un déficit de la $\beta$-oxydation mitochondriale. Cependant, l'identification de deux autres produits accumulés, les acides hydroxy-5. hexanoïque et hydroxy-7-octanoïque, permettait de suspecter un déficit en déshydrogénase des acyl-CoA à chaîne moyenne : ils proviennent de l'oxydation en $\omega$-l dans le réticulum endoplasmique des mêmes intermédiaires de dégradation des acides gras [11]; la prépondérance de l'excrétion de l'acide hydroxy-7-octanoïque par rapport à celle de l'acide hydroxy-5-hexanoïque laissait sup- duit, marqueur du déficit enzymatique au même titre que la subérylglycine [12].

Sans entreprendre une revue exhaustive des maladies métaboliques de l'enfant qu'il est possible de détecter ou d'étudier par spectrométrie de masse, la figure 8, p. 652, donne quelques exemples sur des voies métaboliques aussi diverses que celles des acides aminés, des lipides, ou de la néoglucogenèse. Le plus souvent l'analyse par spectrométrie de masse permet de localiser le déficit enzymatique. Dans certains cas toutefois, elle ne le fournit pas directement, mais est une étape d'orientation diagnostique très importante conduisant à la mesure des activités enzymatiques correspondantes dans le foie et/ou les fibroblastes.

\section{De la molécule au dosage}

La très haute spécificité de la spectrométrie de masse peut être mise à profit pour le dosage précis de métabolites, particulièrement à très faibles concentrations dans le plasma ou le liquide céphalorachidien. L'exemple le plus typique est celui de l'acide phytanique: les valeurs déterminées en chromatographie en phase gazeuse simple sont considérées comme pathologiques lorsqu'elles sont supérieures à 60 $\mu \mathrm{mol} / \mathrm{l}$, alors que la normale plasmatique obtenue en spectrométrie de masse est inférieure à $3 \mu \mathrm{mol} / \mathrm{l}[13]$. Cette discordance peut entraîner des erreurs de diagnostic par défaut quand l'acide phytanique est dosé par chromatographie en phase gazeuse seule. Cela n'a pas d'incidence particulière pour l'établissement du diagnostic de la maladie de Refsum adulte (déficit en phytanate oxydase), où les valeurs sont habituellement de l'ordre de 150 à 600 $\mu \mathrm{mol} / \mathrm{l}$, mais devient crucial dans le cas des maladies peroxysomiales de l'enfant, où les valeurs les plus pathologiques ne dépassent pas 50 $4 \mathrm{~mol} / \mathrm{l}[6]$.

Ce type de dosage nécessite la comparaison du produit à doser avec un «standard interne» qui est le plus souvent un analogue de structure. Toutefois, dans certains cas comme celui de l'acide pipécolique ou l'acide glutarique, il est nécessaire d'utiliser la technique de «dilution 
isotopique » par addition aux échantillons d'une quantité définie des mêmes produits marqués par ${ }^{13} \mathrm{C}$ et/ou par ${ }^{15} \mathrm{~N}$. Ces standards internes ont strictement le même comportement chimique que les produits naturels au cours de l'extraction et de la dérivation, et le rapport obtenu entre le produit naturel et celui marqué par ${ }^{13} \mathrm{C}$ et par ${ }^{15} \mathrm{~N}$ permet d'obtenir de façon très précise la concentration du composé naturel.

\section{Application au diagnotic anténatal}

En raison de ses qualités de sensibilité et de spécificité, la spectrométrie de masse est aussi devenue une méthode de choix pour le diagnostic anténatal de certaines maladies métaboliques. C'est le cas de l'acidémie isovalérique [14], de l'acidémie propionique [15], de l'acidémie méthylmalonique [16], des aciduries glutariques de type I[17] et II [18], de la tyrosinémie de type I [19] et des maladies peroxysomiales [20]; le diagnostic anténatal de ce dernier groupe de maladies peut être réalisé par une analyse par chromatographie en phase gazeuse des acides gras à très longue chaîne obtenus à partir des amniocytes. Il faut alors mettre ceuxci en culture pour obtenir une quantité suffisante d'acides gras cellulaires permettant l'analyse. La spectrométrie de masse rend le diagnostic plus facile et plus rapide : il suffit en effet d'une faible quantité (5 à $10 \mathrm{mg}$ ) de villosités choriales prélevées entre 10 et 12 semaines de grossesse (figure 9) dont on mesure directement le contenu en acide hexacosanoïque [21].

La spectrométrie de masse permet aussi de reconnaître les patientes hétérozygotes pour l'adrénoleucodystrophie liée au chromosome $\mathrm{X}$ qui ont une élévation modérée des acides gras plasmatiques à très longue chaîne (figure 9) [22], ce qui a une répercussion importante au niveau du conseil génétique.

\section{Perspectives}

Nous n'avons abordé ici que les applications du couplage chromatographie en phase gazeuse-spectrométrie de masse. En raison de sa com-

\begin{tabular}{|c|c|}
\hline $\begin{array}{l}\text { Déficits } \\
\text { du métabolisme }\end{array}$ & $\begin{array}{c}\text { Produit(s) accumulé(s) } \\
\text { dans le plasma } \\
\text { etlou l'urine }\end{array}$ \\
\hline \multicolumn{2}{|l|}{ des acides aminés } \\
\hline acidurie 3-hydroxy-3-méthyl-glutarique & acide 3-hydroxy-3-méthyl-glutarique \\
\hline B-méthylcrotonylglycinurie & B-méthylcrotonylglycine \\
\hline acidurie 2-cétoadipique & acide 2-cétoadipique \\
\hline acidémie isovalérique & isovalérylglycine \\
\hline tyrosinémie de type I & succinylacétone \\
\hline déficience en B-cétothiolase & $\begin{array}{l}\text { acides méthyl-2-hydroxy-3-butyrique et } \\
\text { méthyl-2-céto-3-butyrique, tiglyl-glycine }\end{array}$ \\
\hline acidémie $\mathrm{N}$-acétyl-aspartique & acide $\mathrm{N}$-acétyl-aspartique \\
\hline \multicolumn{2}{|l|}{ des lipides } \\
\hline déficiences en camitine & acides dicarboxyliques \\
\hline maladie peroxysomiales & $\begin{array}{l}\text { acides gras à très longue chaîne } \\
\text { acide phytanique } \\
\text { acides biliaires } \\
\text { acide pipécolique } \\
\text { acides dicarboxyliques }\end{array}$ \\
\hline acidémie glutarique type I et II & acide glutarique \\
\hline aciduries dicarboxyliques & acides dicarboxyliques \\
\hline acidémie éthylmalonique-adipique & acides éthylmalonique et adipique \\
\hline \multicolumn{2}{|l|}{$\begin{array}{l}\text { du cycle de Krebs } \\
\text { et de la néoglucogenèse }\end{array}$} \\
\hline acidurie fumarique & acide fumarique \\
\hline acidurie glycérique & acide glycérique \\
\hline acidémie propionique & acide propionique \\
\hline acidémie méthylmalonique & acide méthylmalonique \\
\hline
\end{tabular}

Figure 8. Exemples de maladies métaboliques détectables par spectrométrie de masse.

rience analytique nécessaire, l'implantation de ce couplage en milieu hospitalier reste encore très limitée ; à notre connaissance, deux ou trois laboratoires français spécialisés dans le domaine des maladies métaboliques de l'enfant sont équipés d'un tel matériel.
Les spectromètres de masse « de paillasse » semblent depuis quelques années s'implanter plus facilement en milieu hospitalier de par leurs prix plus accessibles. Bien que moins performants car ne disposant pas de l'ionisation chimique, ils peuvent néanmoins servir à de nombreux 


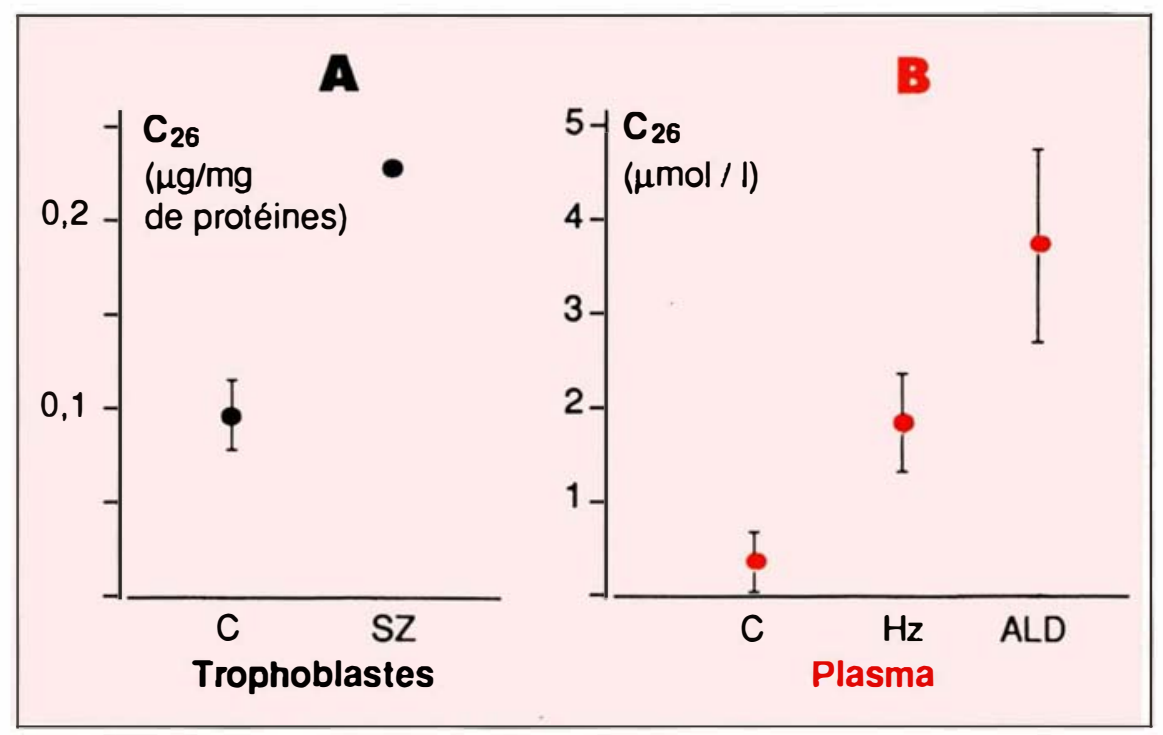

Figure 9. Contenu en acide hexacosanoïque des trophoblastes (A) et du plasma (B) exprimé respectivement en $\mu \mathrm{g} / \mathrm{mg}$ de protéines et en $\mu \mathrm{mol} / \mathrm{I}$. $C$ : contrôles; SZ: fœtus atteint d'un syndrome de Zellweger; $\mathrm{Hz}$ : patientes hétérozygotes pour l'adrénoleucodystrophie liée au chromosome $X$; ALD : patients atteints de l'adrénoleucodystrophie liée au chromosome $X$.

dosages.

Les perspectives les plus prometteuses paraissent réservées aux nouvelles techniques d'ionisation développées depuis peu. La désorption par laser permet de caractériser avec une très grande sensibilité (quelques centaines de fmoles) des protéines de poids moléculaire supérieur à 100000 [23]. Le fast atom bombardment (FAB) permet de mesurer très rapidement, et sans nécessité de purification importante ni de dérivation de l'échantillon, le poids moléculaire de composés polaires thermolabiles non volatils ou de composés à haut poids moléculaire. Cette technique s'est récemment révélée particulièrement utile pour l'analyse des acylcarnitines qui s'accumulent dans les maladies de la $\beta$-oxydation mitochondriale [24]. Le couplage en tandem de trois quadripôles (tandem MS/MS) représente un niveau encore supérieur de complexité analytique. Cette technique a une spécificité encore supérieure à celle de la spectrométrie de masse simple avec une sensibilité égale, ce qui peut permettre d'éliminer l'étape chromatographique. Elle donne également plus d'informations en moins de temps

$\mathrm{m} / \mathrm{s} n^{\circ} 9$ vol. 5, novembre 89

\section{Summary}

Mass spectrometry and inherited metabolic diseases

Mass spectrometry, an established sensitive and specific tool for the structural characterization and quantitation of organic molecules, has today many important developments in the management of the inherited metabolic diseases. The extended range of organic molecules that can be analysed allows to localize the primary enzyme defect in many situations. His remarkable sensitivity is also particularly valuable for precise quantitation of abnormal compounds and finds direct application in the prenatal diagnosis of several metabolic disorders.

\section{TIRÉS A PART}

F. Rocchiccioli. 